\title{
AN EXTENDED LOOMIS-WHITNEY INEQUALITY FOR POSITIVE DOUBLE JOHN BASES
}

\author{
AI-JUN LI \\ School of Mathematics and Information Science, Henan Polytechnic University, Jiaozuo City 454000, China \\ e-mail: liaijun72@163.com \\ GUANGTING WANG \\ Department of Mathematics, Shanghai University, Shanghai 200444, China \\ e-mail: tinggw@163.com \\ and GANGSONG LENG \\ Department of Mathematics, Shanghai University, Shanghai 200444, China \\ e-mail: gleng@staff.shu.edu.cn \\ (Received 27 January 2010; revised 16 August 2010; accepted 21 September 2010; \\ first published online 10 March 2011)
}

\begin{abstract}
In this paper, we establish an extended Loomis-Whitney inequality for positive double John bases, which generalises Ball's result [1]. Moreover, a different extension of the Loomis-Whitney inequality is deduced.
\end{abstract}

2010 Mathematics Subject Classification. 52A20, 52A21, 52A40.

1. Introduction. A convex body $K$ (i.e. compact, convex sets with non-empty interior) in $\mathbb{R}^{n}$ is in John's position if the maximal volume ellipsoid of $K$ is the Euclidean unit ball. John $[\mathbf{3}, \mathbf{1 1}]$ proved that a convex body $K$ is in John's position if and only if there exist contact points $\bar{u}_{1}, \ldots, \bar{u}_{m}$ of $K$ and $B_{2}^{n}$ (common points of their boundaries) and positive real numbers $c_{1}, \ldots, c_{m}$ such that

$$
\sum_{i=1}^{m} c_{i} \bar{u}_{i}=0 \quad \text { and } \quad I_{n}=\sum_{i=1}^{m} c_{i} \bar{u}_{i} \otimes \bar{u}_{i}
$$

where $\bar{u}_{i} \otimes \bar{u}_{i}$ is the usual rank one orthogonal projection onto the span of $\bar{u}_{i}$ and $I_{n}$ is the identity on $\mathbb{R}^{n}$. The first condition guarantees that the $\left\{\bar{u}_{i}\right\}_{1}^{m}$ do not all lie on one side of the sphere. The second condition guarantees that the $\left\{\bar{u}_{i}\right\}_{1}^{m}$ do not all lie close to a proper subspace of $\mathbb{R}^{n}$. In the case of a symmetric convex body, the first condition is redundant, since we can take any sequence $\left\{\bar{u}_{i}\right\}$ of contact points satisfying the second condition and replace each $\bar{u}_{i}$ by the pair $\pm \bar{u}_{i}$, each with half the weight of the original.

The authors would like to acknowledge the support from the National Natural Science Foundation of China (10971128), Shanghai Leading Academic Discipline Project (S30104) and Scientific Research and Innovation Project of Shanghai Municipal Education Commission (09ZZ94). 
The above identity states that the $\bar{u}_{i}$ 's are distributed rather like an orthonormal basis in the sense that for each $x \in \mathbb{R}^{n}$,

$$
|x|^{2}=\sum_{i=1}^{m} c_{i}\left\langle\bar{u}_{i}, x\right\rangle^{2}, \quad \sum_{i=1}^{m} c_{i}=n,
$$

where $\langle\cdot, \cdot\rangle$ is the Euclidean scalar product.

DeFINITION 1. Let $\left\{\bar{u}_{i}\right\}_{1}^{m}$ be a sequence of unit vectors in $\mathbb{R}^{n}$. We call $\left\{\bar{u}_{i}\right\}_{1}^{m}$ a John basis with weights $c_{1}, \ldots, c_{m}>0$, if

$$
I_{n}=\sum_{i=1}^{m} c_{i} \bar{u}_{i} \otimes \bar{u}_{i} .
$$

Note that the condition (1.1) guarantees $m \geq n$.

In fact, John's decomposition of the identity holds in a much more general context. We refer to $[3,4,7,8,9,12,15,17]$ and references therein for an extensive survey of John's decomposition.

In particular, Giannopoulos et al. [7] provided a generalisation of John's representation of the identity for the maximal volume position of two arbitrary smooth convex bodies. This remarkable work can be stated as follows. Let $K, L$ be two (not necessarily symmetric) smooth convex bodies in $\mathbb{R}^{n}$. We say that $L$ is of maximal volume in $K$ if $L \subseteq K$ and, for every $w \in \mathbb{R}^{n}$ and $T \in S L(n)$, the affine image $w+T(L)$ of $L$ is not contained in the interior of $K$.

THEOREM. If $L$ is of maximal volume in $K$, then for every $z$ belonging to the interior of $L$, we can find contact points $v_{1}, \ldots, v_{m}$ of $K-z$ and $L-z$, contact points $u_{1}, \ldots, u_{m}$ of $(K-z)^{\circ}$ and $(L-z)^{\circ}$, and positive real numbers $c_{1}, \ldots, c_{m}$, such that $\sum c_{i} u_{i}=0$, $\left\langle u_{i}, v_{i}\right\rangle=1$, and

$$
I_{n}=\sum_{i=1}^{m} c_{i} u_{i} \otimes v_{i}
$$

Here $K^{\circ}$ is the polar body of $K$, defined by

$$
K^{\circ}=\left\{x \in \mathbb{R}^{n}:\langle x, y\rangle \leq 1 \text { for all } y \in K\right\} .
$$

As usual, $u_{i} \otimes v_{i}$ denotes the rank one projection defined by $u_{i} \otimes v_{i}(x)=\left\langle u_{i}, x\right\rangle v_{i}$. Moreover, there exists a choice of $z$ such that we simultaneously have $\sum c_{i} u_{i}=\sum c_{i} v_{i}=$ 0 . This automatically holds for two symmetric convex bodies.

It is easy to verify from (1.2) that for each $x \in \mathbb{R}^{n}$

$$
|x|^{2}=\sum_{i=1}^{m} c_{i}\left\langle u_{i}, x\right\rangle\left\langle v_{i}, x\right\rangle
$$

and

$$
\sum_{i=1}^{m} c_{i}=n
$$


Motivated by the result of Giannopoulos et al. [7], we give the following definition.

Definition 2. Let $u_{i}, v_{i} \in \mathbb{R}^{n}, i=1, \ldots, m$. We call the sequence of pairs $\left\{\left(u_{i}, v_{i}\right)\right\}_{1}^{m}$ a double John basis with weights $c_{1}, \ldots, c_{m}>0$ if

(i) $\left\langle u_{i}, v_{i}\right\rangle=1$,

(ii) $I_{n}=\sum_{i=1}^{m} c_{i} u_{i} \otimes v_{i}$.

Let $I \subseteq\{1,2, \ldots, m\}$. Denote by $|I|$ its cardinality.

Definition 3. A double John basis $\left\{\left(u_{i}, v_{i}\right)\right\}_{1}^{m}$ is said to be a positive double John basis if it satisfies

$$
\operatorname{det}\left(\sum_{i \in I,|I|=n} u_{i} \otimes v_{i}\right) \geq 0
$$

In this paper, by using positive double John bases as defined above, we will establish an extension of the well-known Loomis-Whitney inequality.

The well-known Loomis-Whitney inequality (see [13] and [5, p. 95]) states that for a convex body $K$ in $\mathbb{R}^{n}$ and a canonical orthonormal basis $\left\{e_{i}\right\}_{1}^{n}$, we have

$$
V(K)^{n-1} \leq \prod_{i=1}^{n} V_{n-1}\left(P_{e_{i}} K\right)
$$

where $P_{e_{i}} K$ is the projection of $K$ onto the 1-codimensional subspace $e_{i}^{\perp}$ orthogonal to $e_{i}$.

The remarkable fact that the orthonormal basis in the above inequality can be replaced by any John basis was established by Ball [1]. Using induction, Ball gave an elegant proof of the following result: If $K$ is a convex body in $\mathbb{R}^{n}$, and $\left(\bar{u}_{1}, \ldots, \bar{u}_{m}\right)$ is a John basis with weights $c_{1}, \ldots, c_{m}>0$, then

$$
V(K)^{n-1} \leq \prod_{i=1}^{m} V_{n-1}\left(P_{\bar{u}_{i}} K\right)^{c_{i}} .
$$

Using a slightly different method than Ball, we establish the following generalisation of inequality (1.6).

THEOREM 1.1. Let $K$ be a convex body in $\mathbb{R}^{n}$. If $\left\{\left(u_{i}, v_{i}\right)\right\}_{1}^{m}$ is a positive double John basis with weights $c_{1}, \ldots, c_{m}>0$, then

$$
V(K)^{2(n-1)} \leq \prod_{i=1}^{m}\left(\left|u_{i}\right|\left|v_{i}\right| V_{n-1}\left(P_{u_{i}} K\right) V_{n-1}\left(P_{v_{i}} K\right)\right)^{c_{i}} .
$$

From an application of Theorem 1.1, we give a different extension of the LoomisWhitney inequality, which generalises Zhang's result [18]. 
THEOREM 1.2. Let $K$ be a convex body in $\mathbb{R}^{n}$. If $\left\{\left(u_{i}, v_{i}\right)\right\}_{1}^{m}$ is a sequence of pair of non-zero vectors in $\mathbb{R}^{n}$ such that

$$
\operatorname{det}\left(\sum_{i \in I,|I|=n} u_{i} \otimes v_{i}\right) \geq 0
$$

and $\sum_{i=1}^{m} u_{i} \otimes v_{i}:=A$ is a positive definite matrix, then

$$
V(K)^{2(n-1)} \leq \operatorname{det}\left(\frac{A+A^{T}}{2}\right)^{-1} \prod_{i=1}^{m}\left(\frac{\left|u_{i}\right|\left|v_{i}\right| V_{n-1}\left(P_{u_{i}} K\right) V_{n-1}\left(P_{v_{i}} K\right)}{c_{i}}\right)^{c_{i}}
$$

where $c_{i}=\left\langle\left(\frac{A+A^{T}}{2}\right)^{-1} u_{i}, v_{i}\right\rangle$.

The rest of this paper is organised as follows: In Section 2 some of the basic notations and preliminaries are established. Section 3 contains the proofs of the main results.

2. Notations and Preliminaries. For $K$ and $L$ convex bodies in $\mathbb{R}^{n}$ and $\lambda \in \mathbb{R}$, the Minkowski sum $K+L$ of $K$ and $L$ is defined by

$$
K+L=\{x+y ; x \in K, y \in L\},
$$

and the scalar multiplication $\lambda K$ is defined by

$$
\lambda K=\{\lambda x ; x \in K\} .
$$

The Minkowski sum of finitely many line segments is called a zonotope.

As a consequence of Minkowski's theorem (see $[6,16])$, the volume of $K+\lambda L$ can be represented by a polynomial in $\lambda$,

$$
V(K+\lambda L)=\sum_{i=0}^{m}\left(\begin{array}{l}
n \\
i
\end{array}\right) V_{i}(K, L) \lambda^{i}
$$

where

$$
V_{i}(K, L)=V(\underbrace{K, \ldots, K}_{n-i}, \underbrace{L, \ldots, L}_{i})
$$

is called the $i$ th mixed volume of $K$ and $L$, where $K$ appears $n-i$ times and $L$ appears $i$ times. The Brunn-Minkowski inequality states that $V(K+\lambda L)^{1 / n}$ is a concave function of $\lambda$ in $[0, \infty)$. Differentiation of $(2.1)$ at $\lambda=0$ gives Minkowski's first inequality

$$
V_{1}(K, L) \geq V(K)^{(n-1) / n} V(L)^{1 / n}
$$

with equality if and only if $K$ and $L$ are homothetic. By Cauchy's projection formula [6], we can easily obtain that if $\bar{u}$ is a unit vector then

$$
V_{1}(K,[-\bar{u}, \bar{u}])=\frac{2}{n} V_{n-1}\left(P_{\bar{u}_{i}} K\right)
$$


for any convex body $K$. Let $\left[-u_{1}, u_{1}\right], \ldots,\left[-u_{m}, u_{m}\right]$ be $m$ line segments, their Minkowski sum is

$$
Z=\left[-u_{1}, u_{1}\right]+\cdots+\left[-u_{m}, u_{m}\right]
$$

So

$$
\begin{aligned}
V_{1}(K, Z) & =V_{1}\left(K, \sum_{i=1}^{m}\left[-u_{i}, u_{i}\right]\right)=V_{1}\left(K, \sum_{i=1}^{m}\left|u_{i}\right|\left[-\bar{u}_{i}, \bar{u}_{i}\right]\right) \\
& =\frac{2}{n} \sum_{i=1}^{m}\left|u_{i}\right| V_{n-1}\left(P_{\bar{u}_{i}} K\right)=\frac{2}{n} \sum_{i=1}^{m}\left|u_{i}\right| V_{n-1}\left(P_{u_{i}} K\right),
\end{aligned}
$$

where $\bar{u}_{i}=u_{i} /\left|u_{i}\right|$. If $u_{1}, \ldots, u_{m} \in \mathbb{R}^{n}, m \geq n$, we have (see [14, p. 73])

$$
V\left(\sum_{i=1}^{m}\left[-u_{i}, u_{i}\right]\right)=2^{n} \sum_{1 \leq i_{1}<\cdots<i_{n} \leq m}\left|\operatorname{det}\left(u_{i_{1}}, \ldots, u_{i_{n}}\right)\right| .
$$

In order to prove Theorem 1.1, it should be noted that if $p>0, r_{i}, a_{i}>0$, the weighted $p$ th means $\sum_{i}\left(r_{i} a_{i}^{p}\right)^{1 / p}$ decrease with $p$ for all $a_{i}>0$ if and only if $r_{i} \geq 1$. See [10 (2.10.5), p. 29]. In particular, the inequality

$$
\sum_{i} r_{i} a_{i}^{2} \leq\left(\sum_{i} r_{i} a_{i}\right)^{2}
$$

is true for all $a_{i}>0$ when $r_{i} \geq 1$.

3. Proof of the main results. The Cauchy-Binet formula can be stated as follows.

LEMMA 3.1. Let $m \geq n$ be integers and $I \subseteq\{1,2, \ldots, m\}$. Let $A$ be a $n \times m$ matrix and $B$ a $m \times n$ matrix. If $A_{I}$ denotes the square matrix obtained from $A$ by keeping only the columns with indices in $I$, and $B_{I}$ denotes the square matrix obtained from $B$ by keeping the rows with indies in I, then we have the formula

$$
\operatorname{det}(A B)=\sum_{|I|=n} \operatorname{det}\left(A_{I}\right) \operatorname{det}\left(B_{I}\right)
$$

Using the Cauchy-Binet formula and the fact that $\left\langle u_{i}, v_{i}\right\rangle=1$, the following critical lemma was proved by Giannopoulos et al. [7].

LEMMA 3.2. If a sequence of pairs $\left\{\left(u_{i}, v_{i}\right)\right\}_{1}^{m}$ is a positive double John basis, with weights $c_{1}, \ldots, c_{m}>0$, then for $\lambda_{i}, \delta_{i}>0, i=1, \ldots, m$, we have

$$
\operatorname{det}\left(\sum_{i=1}^{m} c_{i} \lambda_{i} u_{i} \otimes u_{i}\right) \operatorname{det}\left(\sum_{i=1}^{m} c_{i} \delta_{i} v_{i} \otimes v_{i}\right) \geq \prod_{i=1}^{m}\left(\lambda_{i} \delta_{i}\right)^{c_{i}} .
$$

Proof. Let $I \subseteq\{1,2, \ldots, m\}$. Write $\lambda_{I}=\prod_{i \in I} \lambda_{i}, \quad \delta_{I}=\prod_{i \in I} \delta_{i}$ and use the notations $U_{I}=\operatorname{det}\left(u_{i} ; i \in I\right), V_{I}=\operatorname{det}\left(v_{i} ; i \in I\right)^{T}$, where $T$ is the notation of transpose. Moreover, we write $(\sqrt{c} U)_{I}$ for $\operatorname{det}\left(\sqrt{c_{i}} u_{i} ; i \in I\right)$ and $(\sqrt{c} V)_{I}$ for $\operatorname{det}\left(\sqrt{c_{i}} v_{i} ; i \in I\right)^{T}$. 
Applying the Cauchy-Binet formula, we obtain

$$
1=\operatorname{det} I_{n}=\operatorname{det}\left(\sum_{i=1}^{m} c_{i} u_{i} \otimes v_{i}\right)=\sum_{\substack{|I|=n \\ I \subseteq\{1,2, \ldots, m\}}}(\sqrt{c} U)_{I}(\sqrt{c} V)_{I}
$$

and

$$
\operatorname{det}\left(\sum_{i=1}^{m} c_{i} \lambda_{i} u_{i} \otimes v_{i}\right)=\sum_{\substack{\mid I I=n \\ I \subseteq\{1,2, \ldots, m\}}} \lambda_{I}(\sqrt{c} U)_{I}(\sqrt{c} V)_{I}
$$

Being a positive double John basis $\left\{\left(u_{i}, v_{i}\right)\right\}_{1}^{m}$, it is easy to verify that

$$
\operatorname{det}\left(\sum_{i \in I,|I|=n} u_{i} \otimes v_{i}\right)=U_{I} V_{I} \geq 0
$$

This guarantees that the coefficients $(\sqrt{c} U)_{I}(\sqrt{c} V)_{I}$ are all non-negative. Then applying the arithmetic-geometric means inequality [10] with coefficients $(\sqrt{c} U)_{I}(\sqrt{c} V)_{I}$, we get

$$
\begin{aligned}
\sum_{\substack{|I|=n \\
I \subseteq\{1,2, \ldots, m\}}} \lambda_{I}(\sqrt{c} U)_{I}(\sqrt{c} V)_{I} & \geq \prod_{\substack{|I|=n \\
I \subseteq\{1,2, \ldots, m\}}} \lambda_{I}^{(\sqrt{c} U)_{I}(\sqrt{c} V)_{I}} \\
& =\prod_{j=1}^{m} \lambda_{j}^{\sum_{j \in I,|I|=n}(\sqrt{c} U)_{I}(\sqrt{c} V)_{I} .} .
\end{aligned}
$$

Observe that

$$
\begin{aligned}
\sum_{j \in I,|I|=n}(\sqrt{c} U)_{I}(\sqrt{c} V)_{I} & =\sum_{|I|=n}(\sqrt{c} U)_{I}(\sqrt{c} V)_{I}-\sum_{j \notin I,|I|=n}(\sqrt{c} U)_{I}(\sqrt{c} V)_{I} \\
& =\operatorname{det}\left(\sum_{i=1}^{m} c_{i} u_{i} \otimes v_{i}\right)-\operatorname{det}\left(I_{n}-c_{j} u_{j} \otimes v_{j}\right) \\
& =1-\operatorname{det}\left(I_{n}\right) \operatorname{det}\left(1-c_{j}\left\langle u_{j}, v_{j}\right\rangle\right) \\
& =c_{j},
\end{aligned}
$$

since $\left\langle u_{j}, v_{j}\right\rangle=1$.

Thus, we obtain that

$$
\operatorname{det}\left(\sum_{i=1}^{m} c_{i} \lambda_{i} u_{i} \otimes v_{i}\right) \geq \prod_{i=1}^{m} \lambda_{i}^{c_{i}}
$$


By the Cauchy-Schwarz inequality, we have

$$
\begin{aligned}
& \operatorname{det}\left(\sum_{i=1}^{m} c_{i} \lambda_{i} u_{i} \otimes u_{i}\right) \operatorname{det}\left(\sum_{i=1}^{m} c_{i} \delta_{i} v_{i} \otimes v_{i}\right) \\
& =\sum_{\substack{|I|=n \\
I \subseteq\{1,2, \ldots, m\}}} \lambda_{I}(\sqrt{c} U)_{I}^{2} \sum_{\substack{|I|=n \\
I \subseteq\{1,2, \ldots, m\}}} \delta_{I}(\sqrt{c} V)_{I}^{2} \\
& \geq\left(\sum_{\substack{\mid[I \mid=n \\
I \subseteq\{1,2, \ldots, m\}}} c_{I} \sqrt{\lambda_{I} \delta_{I}} U_{I} V_{I}\right)^{2} .
\end{aligned}
$$

Then applying (3.3), we deduce (3.1), and complete the proof.

REMARK 1. Lemma 3.2 is a revisional version of the original Proposition 4.4 in [7]. In the original proof, the coefficients $(\sqrt{c} U)_{I}(\sqrt{c} V)_{I}$ are not necessarily positive and we can find a counter example. For example, in $\mathbb{R}^{2}$, suppose $m=3$. Let $u_{1}=(1,2), u_{2}=$ $\left(\frac{3}{2}, \frac{1}{2}\right), u_{3}=\left(-1, \frac{18}{161}\right), v_{1}=\left(\frac{1}{2}, \frac{1}{4}\right), v_{2}=\left(-\frac{1}{3}, 3\right), v_{3}=\left(-\frac{161}{194}, \frac{1771}{1164}\right)$ and $c_{1}=\frac{1}{5}, c_{2}=\frac{96}{215}$, $c_{3}=\frac{291}{215}$. It is easy to check that they satisfy $\sum_{i=1}^{3} c_{i} u_{i} \otimes v_{i}=I_{n}, c_{1}+c_{2}+c_{3}=2$ and $\left\langle u_{i}, v_{i}\right\rangle=1, i=1,2,3$. But $\operatorname{det}\left(u_{1}, u_{2}\right) \cdot \operatorname{det}\left(v_{1}, v_{2}\right)<0$. If we put $\lambda_{1}=1, \lambda_{2}=1, \lambda_{3}=$ 100 and $\delta_{i}=1, i=1,2,3$, then the inequality of Proposition 4.4 in [7] does not hold. We realise that the condition of the positive coefficients is necessary. In order to complete the proof of Lemma 3.2, the condition (1.5) has to be added to the definition of double John basis. Since

$$
\sum_{|I|=n}(\sqrt{c} U)_{I}(\sqrt{c} V)_{I}=1
$$

the double John basis with the restricted condition $U_{I} V_{I} \geq 0$ always exists. For example, let $u_{1}=\left(1, \frac{1}{2}\right), u_{2}=\left(-1, \frac{1}{2}\right), u_{3}=\left(-1,-\frac{1}{2}\right), v_{1}=\left(\frac{1}{2}, 1\right), v_{2}=\left(-\frac{1}{2}, 1\right), v_{3}=\left(-\frac{1}{2},-1\right)$ and $c_{1}=\frac{1}{2}, c_{2}=1, c_{3}=\frac{1}{2}$. It is easy to check that $\left\{\left(u_{i}, v_{i}\right)\right\}_{1}^{3}$ is a positive double John basis of $\mathbb{R}^{2}$, with weights $c_{1}, c_{2}, c_{3}$.

Proof of Theorem 1.1. Let $I \subseteq\{1,2, \ldots, m\}$. Denote by $|I|$ its cardinality. Write $\alpha_{I}=\prod_{i \in I} \alpha_{i}, \beta_{I}=\prod_{i \in I} \beta_{i}$ and $c_{I}=\prod_{i \in I} c_{i}$. For $\alpha_{i}, \beta_{i}>0, i=1, \ldots, m$, let

$$
Z_{1}=\sum_{i=1}^{m}\left[-\alpha_{i} u_{i}, \alpha_{i} u_{i}\right], \quad Z_{2}=\sum_{i=1}^{m}\left[-\beta_{i} v_{i}, \beta_{i} v_{i}\right]
$$

Then by (2.4), we have

$$
V\left(Z_{1}\right)=2^{n} \sum_{1 \leq i_{1}<\cdots<i_{n} \leq m}\left|\operatorname{det}\left(\alpha_{i_{1}} u_{i_{1}}, \ldots, \alpha_{i_{n}} u_{i_{n}}\right)\right|=2^{n} \sum_{\substack{\mid I I=n \\ I \subseteq\{1,2, \ldots, m\}}} \alpha_{I}\left|\operatorname{det}_{i \in I}\left(u_{i}\right)\right|
$$

and

$$
V\left(Z_{2}\right)=2^{n} \sum_{1 \leq i_{1}<\cdots<i_{n} \leq m}\left|\operatorname{det}\left(\beta_{i_{1}} v_{i_{1}}, \ldots, \beta_{i_{n}} v_{i_{n}}\right)\right|=2^{n} \sum_{\substack{|I I|=n \\ I \leq\{1,2, \ldots, m\}}} \beta_{I}\left|\operatorname{det}_{i \in I}\left(v_{i}\right)\right| .
$$


For sufficiently small $\varepsilon>0$, we take two sequences of positive numbers $\lambda_{i}, \delta_{i}$, $i=1,2, \ldots, m$, such that

$$
c_{I} \lambda_{I}^{\varepsilon}=\prod_{i \in I} c_{i} \lambda_{i}^{\varepsilon} \geq 1, \quad c_{I} \delta_{I}^{\varepsilon}=\prod_{i \in I} c_{i} \delta_{i}^{\varepsilon} \geq 1
$$

Therefore, by inequality (2.5), we have

$$
\begin{aligned}
& \operatorname{det}\left(\sum_{i=1}^{m} c_{i} \lambda_{i} u_{i} \otimes u_{i}\right) \operatorname{det}\left(\sum_{i=1}^{m} c_{i} \delta_{i} v_{i} \otimes v_{i}\right) \\
& =\sum_{\substack{|I|=n \\
I \subseteq\{1,2, \ldots, m\}}} c_{I} \lambda_{I}\left(\operatorname{det}_{i \in I}\left(u_{i}\right)\right)^{2} \sum_{\substack{|I|=n \\
I \subseteq\{1,2, \ldots, m\}}} c_{I} \delta_{I}\left(\operatorname{det}_{i \in I}\left(v_{i}\right)\right)^{2} \\
& =\sum_{\substack{|I|=n \\
I \subseteq\{1,2, \ldots, m\}}}\left(c_{I} \lambda_{I}^{\varepsilon}\right) \lambda_{I}^{1-\varepsilon}\left(\operatorname{det}_{i \in I}\left(u_{i}\right)\right)^{2} \sum_{\substack{|I|=n \\
I \subseteq 1,2, \ldots, m\}}}\left(c_{I} \delta_{I}^{\varepsilon}\right) \delta_{I}^{1-\varepsilon}\left(\operatorname{det}_{i \in I}\left(v_{i}\right)\right)^{2} \\
& \left.\leq\left(\sum_{\substack{|I|=n \\
I \subseteq\{1,2, \ldots, m\}}} c_{I} \lambda_{I}^{\frac{1+\varepsilon}{2}}\left|\operatorname{det}_{i \in I}\left(u_{i}\right)\right|\right)^{2} \sum_{\substack{I I I=n \\
I \subseteq\{1,2, \ldots, m\}}} c_{I} \delta_{I}^{\frac{1+\varepsilon}{2}}\left|\operatorname{det}_{i \in I}\left(v_{i}\right)\right|\right)^{2} \\
& =\left(\frac{V\left(\sum_{i=1}^{m} c_{i} \lambda_{i}^{\frac{1+\varepsilon}{2}}\left[-u_{i}, u_{i}\right]\right)}{2^{n}}\right)^{2}\left(\frac{V\left(\sum_{i=1}^{m} c_{i} \delta_{i}^{\frac{1+\varepsilon}{2}}\left[-v_{i}, v_{i}\right]\right)}{2^{n}}\right)^{2} .
\end{aligned}
$$

Put $c_{i} \lambda_{i}^{\frac{1+\varepsilon}{2}}=\alpha_{i}, c_{i} \delta_{i}^{\frac{1+\varepsilon}{2}}=\beta_{i}$. By (3.4), (3.5) and Lemma 3.2, we obtain that

$$
V\left(Z_{1}\right) V\left(Z_{2}\right) \geq 2^{2 n} \prod_{i=1}^{m}\left(\left(\frac{\alpha_{i}}{c_{i}}\right)^{\frac{2}{1+\varepsilon}}\right)^{\frac{c_{i}}{2}} \prod_{i=1}^{m}\left(\left(\frac{\beta_{i}}{c_{i}}\right)^{\frac{2}{1+\varepsilon}}\right)^{\frac{c_{i}}{2}}=2^{2 n} \prod_{i=1}^{m}\left(\frac{\alpha_{i} \beta_{i}}{c_{i}^{2}}\right)^{\frac{c_{i}}{1+\varepsilon}}
$$

Since the all $c_{i}$ are fixed and $\lambda_{i}, \delta_{i}$ were taken arbitrarily, we can let $\varepsilon \rightarrow 0$ to obtain

$$
V\left(Z_{1}\right) V\left(Z_{2}\right) \geq 2^{2 n} \prod_{i=1}^{m}\left(\frac{\alpha_{i} \beta_{i}}{c_{i}^{2}}\right)^{c_{i}}
$$

Now for each $i$, let

$$
\alpha_{i}=\frac{c_{i}}{\left|u_{i}\right| V_{n-1}\left(P_{u_{i}} K\right)}, \quad \beta_{i}=\frac{c_{i}}{\left|v_{i}\right| V_{n-1}\left(P_{v_{i}} K\right)}
$$


From Minkowski's first inequality (2.2), (2.3) and (1.4), we obtain

$$
\begin{aligned}
V(K)^{2(n-1)} & \leq V\left(Z_{1}\right)^{-1} V\left(Z_{2}\right)^{-1} V_{1}\left(K, Z_{1}\right)^{n} V_{1}\left(K, Z_{2}\right)^{n} \\
& =V\left(Z_{1}\right)^{-1} V\left(Z_{2}\right)^{-1}\left(\frac{2}{n} \sum_{i=1}^{m} \alpha_{i}\left|u_{i}\right| V_{n-1}\left(P_{u_{i}} K\right)\right)^{n}\left(\frac{2}{n} \sum_{i=1}^{m} \beta_{i}\left|v_{i}\right| V_{n-1}\left(P_{v_{i}} K\right)\right)^{n} \\
& =2^{2 n} V\left(Z_{1}\right)^{-1} V\left(Z_{2}\right)^{-1} \\
& \leq 2^{2 n}\left(2^{2 n} \prod_{i=1}^{m}\left(\frac{\alpha_{i} \beta_{i}}{c_{i}^{2}}\right)^{c_{i}}\right)^{-1} \\
& =\prod_{i=1}^{m}\left(\left|u_{i}\right|\left|v_{i}\right| V_{n-1}\left(P_{u_{i}} K\right) V_{n-1}\left(P_{v_{i}} K\right)\right)^{c_{i}} .
\end{aligned}
$$

This completes the proof.

REMARK 2. If $u_{i}=v_{i}$ are unit vectors for all $i$, then the positive double John basis will become a John basis, with weights $c_{1}, \ldots, c_{m}$ and Theorem 1.1 coincides with Ball's result (1.6). In fact, by the Cauchy-Binet formula, (1.1) implies that

$$
1=\operatorname{det} I_{n}=\operatorname{det}\left(\sum_{i=1}^{m} c_{i} u_{i} \otimes v_{i}\right)=\sum_{|I|=n} c_{I} U_{I}^{2} .
$$

Note that the coefficients $U_{I}^{2}$ are always non-negative.

Proof of Theorem 1.2. For $\alpha_{i}, \beta_{i}>0, i=1,2, \ldots, m$, let $Z_{1}=\sum_{i=1}^{m} \alpha_{i}\left[-u_{i}, u_{i}\right]$ and $Z_{2}=\sum_{i=1}^{m} \beta_{i}\left[-v_{i}, v_{i}\right]$. Since $A$ is a positive definite matrix, there exists a non-singular matrix $Q$ such that

$$
Q^{T} Q=\frac{1}{2}\left(A+A^{T}\right)=\frac{1}{2}\left(\sum_{i=1}^{m} u_{i} \otimes v_{i}+\sum_{i=1}^{m} v_{i} \otimes u_{i}\right) .
$$

Let $y=Q x$ for $x \in \mathbb{R}^{n}$. Then

$$
\begin{aligned}
|y|^{2} & =\langle Q x, Q x\rangle=\left\langle\frac{1}{2}\left(A+A^{T}\right) x, x\right\rangle=\frac{1}{2}\langle A x, x\rangle+\frac{1}{2}\left\langle A^{T} x, x\right\rangle \\
& =\frac{1}{2} \sum_{i=1}^{m}\left\langle u_{i}, x\right\rangle\left\langle v_{i}, x\right\rangle+\frac{1}{2} \sum_{i=1}^{m}\left\langle v_{i}, x\right\rangle\left\langle u_{i}, x\right\rangle \\
& =\sum_{i=1}^{m}\left\langle u_{i}, x\right\rangle\left\langle v_{i}, x\right\rangle \\
& =\sum_{i=1}^{m} c_{i}\left\langle\tilde{u}_{i}, y\right\rangle\left\langle\tilde{v}_{i}, y\right\rangle,
\end{aligned}
$$

where $\tilde{u}_{i}=c_{i}^{-\frac{1}{2}} Q^{-T} u_{i}, \tilde{v}_{i}=c_{i}^{-\frac{1}{2}} Q^{-T} v_{i}$ and $c_{i}=\left\langle Q^{-1} Q^{-T} u_{i}, v_{i}\right\rangle$. It follows that

$$
\left\langle\tilde{u}_{i}, \tilde{v}_{i}\right\rangle=c_{i}^{-1}\left\langle Q^{-1} Q^{-T} u_{i}, v_{i}\right\rangle=1
$$


and

$$
\operatorname{det}\left(\sum_{i \in I,|I|=n} \tilde{u}_{i} \otimes \tilde{v}_{i}\right)=c_{I}^{-1} \operatorname{det}\left(Q^{-T}\left(\sum_{i \in I,|I|=n} u_{i} \otimes v_{i}\right) Q^{-1}\right) \geq 0
$$

By the definition of the positive double John basis, (1.3) and (3.7), it is easy to check that $\left\{\left(\tilde{u}_{i}, \tilde{v}_{i}\right)\right\}_{1}^{m}$ is a positive double John basis with weights $c_{1}, \ldots, c_{m}$.

So we have

$$
Z_{1}=\sum_{i=1}^{m} \alpha_{i}\left[-u_{i}, u_{i}\right]=\sum_{i=1}^{m} \alpha_{i}\left[-c_{i}^{\frac{1}{2}} Q^{T} \bar{u}_{i}, c_{i}^{\frac{1}{2}} Q^{T} \bar{u}_{i}\right]=\sum_{i=1}^{m} \alpha_{i} c_{i}^{\frac{1}{2}}\left[-Q^{T} \tilde{u}_{i}, Q^{T} \tilde{u}_{i}\right]
$$

and

$$
Z_{2}=\sum_{i=1}^{m} \beta_{i} c_{i}^{\frac{1}{2}}\left[-Q^{T} \tilde{v}_{i}, Q^{T} \tilde{v}_{i}\right]
$$

Multiplying $Q^{-T}$ on both sides leads to

$$
Q^{-T} Z_{1}=\sum_{i=1}^{m} \alpha_{i} c_{i}^{\frac{1}{2}}\left[-\tilde{u}_{i}, \tilde{u}_{i}\right]
$$

and

$$
Q^{-T} Z_{2}=\sum_{i=1}^{m} \beta_{i} c_{i}^{\frac{1}{2}}\left[-\tilde{v}_{i}, \tilde{v}_{i}\right]
$$

By (3.6), we obtain

$$
\operatorname{det}\left(Q^{-T}\right)^{2} V\left(Z_{1}\right) V\left(Z_{2}\right) \geq 2^{2 n} \prod_{i=1}^{m}\left(\frac{\alpha_{i} c_{i}^{\frac{1}{2}} \beta_{i} c_{i}^{\frac{1}{2}}}{c_{i}^{2}}\right)^{c_{i}}=2^{2 n} \prod_{i=1}^{m}\left(\frac{\alpha_{i} \beta_{i}}{c_{i}}\right)^{c_{i}}
$$

Noticing $\operatorname{det}\left(\frac{A+A^{T}}{2}\right)=\left(\operatorname{det} Q^{T}\right)^{2}$, it follows that

$$
V\left(Z_{1}\right) V\left(Z_{2}\right) \geq 2^{2 n} \operatorname{det}\left(\frac{A+A^{T}}{2}\right) \prod_{i=1}^{m}\left(\frac{\alpha_{i} \beta_{i}}{c_{i}}\right)^{c_{i}}
$$

For each $i$, let

$$
\alpha_{i}=\frac{c_{i}}{\left|u_{i}\right| V_{n-1}\left(P_{u_{i}} K\right)}, \quad \beta_{i}=\frac{c_{i}}{\left|v_{i}\right| V_{n-1}\left(P_{v_{i}} K\right)}
$$


From (2.2), (2.3) and (1.4), we have

$$
\begin{aligned}
V(K)^{2(n-1)} & \leq V\left(Z_{1}\right)^{-1} V_{1}\left(K, Z_{1}\right)^{n} V\left(Z_{2}\right)^{-1} V_{1}\left(K, Z_{2}\right)^{n} \\
& =V\left(Z_{1}\right)^{-1} V\left(Z_{2}\right)^{-1}\left(\frac{2}{n} \sum_{i=1}^{m} \alpha_{i}\left|u_{i}\right| V_{n-1}\left(P_{u_{i}} K\right)\right)^{n}\left(\frac{2}{n} \sum_{i=1}^{m} \beta_{i}\left|v_{i}\right| V_{n-1}\left(P_{v_{i}} K\right)\right)^{n} \\
& =2^{2 n} V\left(Z_{1}\right)^{-1} V\left(Z_{2}\right)^{-1} \\
& \leq 2^{n}\left(2^{2 n} \operatorname{det}\left(\frac{A+A^{T}}{2}\right) \prod_{i=1}^{m}\left(\frac{\alpha_{i} \beta_{i}}{c_{i}}\right)^{c_{i}}\right)^{-1} \\
& =\operatorname{det}\left(\frac{A+A^{T}}{2}\right)^{-1} \prod_{i=1}^{m}\left(\frac{\left|u_{i}\right|\left|v_{i}\right| V_{n-1}\left(P_{u_{i}} K\right) V_{n-1}\left(P_{v_{i}} K\right)}{c_{i}}\right)^{c_{i}} .
\end{aligned}
$$

This gives the result.

REMARK 3. The case of $u_{i}=v_{i}$ for each $i$ was proved by Zhang [18]. If $m=n$ and $u_{i}=v_{i}=e_{i}$, we obtain the classical Loomis-Whitney inequality.

AcKnOwLEDGEMENTS. The authors are indebted to the referee for the valuable suggestions and the very careful reading of the original manuscript.

\section{REFERENCES}

1. K. Ball, Shadows of convex bodies, Trans. Amer. Math. Soc. 327 (1991), 891-901.

2. K. Ball, Volume ratios and a reverse isoperimetric inequality, J. Lond. Math. Soc. 44 (1991), 351-359.

3. K. Ball, An elementary introduction to modern convex geometry, Flavors of geometry, in Mathematical Science Research Institute Publication, Vol. 31 (Levy S., Editor) (Cambridge University Press, Cambridge, UK, 1997), 1-58.

4. J. Bastero and M. Romance, John's decomposition of the identity in the non-convex case, Positivity 6 (2002), 1-161.

5. Yu. D. Burago and V. A. Zalgaller, Geometric inequalities (Springer, Berlin, 1988). 1995).

6. R. J. Gardner, Geometric tomography (Cambridge University Press, Cambridge, UK,

7. A. Giannopoulos, I. Perissinaki and A. Tsolomitis, John's theorem for an arbitrary pair of convex bodies, Geom. Dedicata 84 (2001), 63-79.

8. Y. Gordon, A. E. Litvak, M. Meyer and A. Pajor, John's decomposition in the general case and applications, J. Differ. Geom. 68(1) (2004), 99-119.

9. P. M. Gruber and F. Schuster, An arthmetic proof of John's ellipsoid theorem, Arch. Math. 85 (2005), 82-88.

10. G. H. Hardy, J. E. Littlewood and G. Pólya, Inequalities (Cambridge University Press, Cambridge, UK, 1959).

11. F. John, Extremum problems with inequalities as subsidiary conditions, in Studies and essays presented to R. Courant on his 60th birthday, January 8, 1948 (Interscience Publishers, New York, NY, 1948), 187-204.

12. D. Lewis, Ellipsoids defined by Banach ideal norms, Mathematika 26 (1979), 18-29.

13. L. H. Loomis and H. Whitney, An inequality related to the isoperimetric inequality, Bull. Amer. Math. Soc. 55 (1949), 961-962.

14. V. D. Milman and A. Pajor, Isotropic position and inertia ellipsoids and zonoids of the unit ball of a normed n-dimensional space, in Geometric aspects of functional analysis (Lindenstrauss-Milman, Editor), Lecture Notes in Math., 1376 (Springer, Berlin, 1989), 64 104.

15. G. Pisier, The volume of convex bodies and Banach space geometry, in Cambridge tracts in mathematics, Vol. 94, (Cambridge University Press, Cambridge, UK, 1989). 
16. R. Schneider, Convex bodies: The Brunn-Minkowski theory, in Encyclopedia of mathematics and its applications, Vol. 44, (Cambridge University Press, Cambridge, UK, 1993).

17. N. Tomczak-Jaegermann, Banach-Mazur distances and finite-dimensional operator ideals, in Pitman monographs and surveys in pure and applied mathematics, Vol. 38 (Pitman, London, 1989).

18. G. Zhang, The affine Sobolev inequality, J. Differ. Geom. 53 (1999), 183-202. 